\title{
Computed Transient Beam Loading Effects with Harmonic RF Systems *
}

\author{
J. M. Byrd, S. De Santis, LBNL, Berkeley, California \\ J. Jacob, V. Serrière, ESRF, Grenoble, France
}

\begin{abstract}
Harmonic cavities have been used in storage rings to lengthen bunches and increase beam lifetimes dominated by Touschek scattering. Transient beam loading in the harmonic cavities generated by asymmetries in the fill pattern can cause significant variation of the beam phase and bunch length along the bunch train when the longitudinal restoring force has been reduced, resulting in a significant reduction in the mean bunch lengthening and potential lifetime increase. We describe how beam current modulations give rise to transient effects much larger than expected from the linear model of the beam-cavity interaction. We also develop a tracking simulation to predict results and apply this simulation to an analysis of the beam loading transients for the case of a passive and active normal and superconducting third harmonic RF systems using ALS parameters.
\end{abstract}

\section{INTRODUCTION}

Harmonic RF systems have recently been installed and commissioned on several third generation light sources. In these rings, the distribution of current in RF buckets is rarely uniform because of limitations in the injector, gaps for beam kicker rise times or for clearing trapped ions, or because of user requirements. We have observed in the ALS that gaps in the fill pattern create transient beam loading effects along the bunch train which significantly degrade the total amount of bunch lengthening and thus the lifetime improvement. There is an additional side effect of a large variation in beam synchronous phase along the bunch train, causing problems for a number of technical systems. We believe that the transient effects present a serious limitation to the lifetime improvement which can be achieved with harmonic systems in third generation light sources, regardless of whether the system is active or passive, normal or superconducting.

\section{TRANSIENTS IN HARMONIC RF SYSTEM}

Transient beam loading in storage ring RF systems has been studied for some times. F. Pedersen's small-signal model of the beam-cavity interaction gives an accurate method to compute transient beam loading effects [1]. However, for describing the transient effects with harmonic cavities, the Pedersen model is lacking in two respects. Firstly, the harmonic voltage strongly affects the beam transfer function by inducing a spread in synchrotron frequencies [2], thereby violating the assumption of linear

\footnotetext{
* This work was supported by the U.S. Department of Energy under Contract Nos. DE-AC03-76SF00098.
}

transfer functions. Secondly, the model applies only to small modulations of the steady state values. As shown in section 3 this assumption is also not valid in the case of a harmonic RF system. To further study the transient effects in the absence of an analytical model, we have developed a computer tracking code to find the steady state harmonic voltage and phase along the bunch train [3]. Using this, we calculate the corresponding bunch shape and lifetime increase for each bunch and the overall lifetime increase of the beam.

\section{EXAMPLES OF COMPUTER SIMULATION.}

In this section we present several examples computed with the tracking code using ALS parameters. Five normal conducting (NC) third harmonic cavities have been installed at the ALS . However, due to the Robinson effect, only up to three cavities can be operated in bunch lengthening mode. The tracking code shows that the best improvement in lifetime is given for three harmonic cavities tuned at $h_{\text {res }}=f_{\text {res }} / f_{0}=984.32$ where $f_{\text {res }}$ is the resonant frequency of the harmonic cavity anf $f_{0}$ the revolution frequency. In case of a perfectly uniform fill with $400 \mathrm{~mA}$ average beam current, the tracking code shows that all the bunches lengthen by the same factor of 2.2, in agreement with the analytical calculation [2]. For the same current with a $17 \%$ gap in the fill pattern, as commonly operated at ALS, the tracking simulation yields the transient effects depicted in Figure 1. In this case, the beam phase converges to a different value for each bunch. The steadystate distribution of the synchronous phase along the bunch train in Figure 1a shows a total phase transient of about 0.3 $\mathrm{rad}$. The modulation of the harmonic voltage plotted in Figure $1 \mathrm{~b}$, reaching $\pm 17 \%$ in amplitude and $\pm 0.6 \mathrm{rad}$ in phase, leads to a variation in the bunch lengthening along the train. As shown in Figure 1c the maximum bunch lengthening of about 1.46 for the fill with gap is lower than the value of 2.2 obtained under similar operation conditions for uniform filling. The average bunch lengthening is less than 1.4 and bunches at the edge of the fill experience a stretching of only 1.32 .

\section{RESULTS}

\subsection{Passive and active harmonic cavities}

We have applied the tracking code to compute the transient effects for various harmonic cavity technologies. For each case, passive operation using the beam to drive the harmonic voltage has been compared to active operation with 

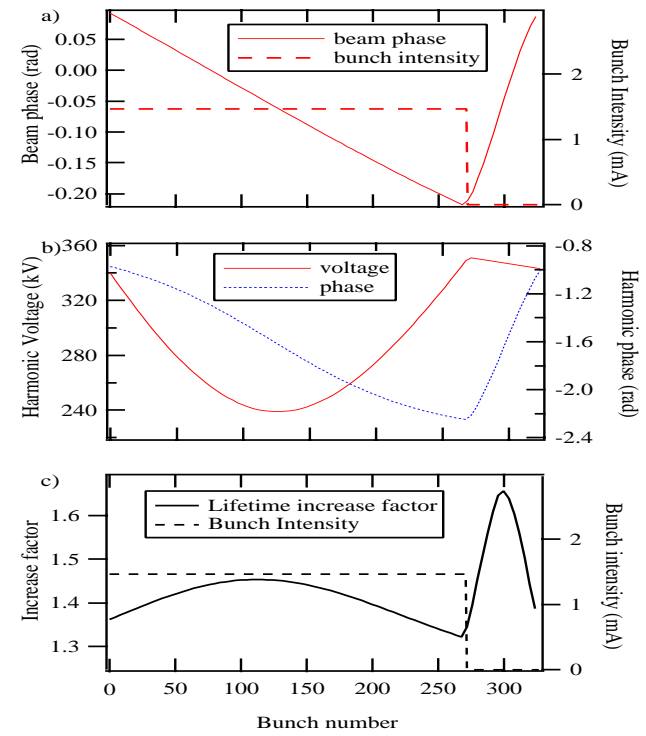

Figure 1: a) Beam phase (difference from nominal synchronous phase) and bunch intensity along the bunch train. b) Harmonic voltage and phase. c) Resulting lifetime increase

an external harmonic generator. The following three different harmonic cavity technologies have been investigated:

- normal conducting (NC) ALS cavity $(R / Q=80.9 \Omega$ and $Q=21000$ ) [4],

- superconducting (SC) super-3HC cavity $(R / Q=$ $87 \Omega$ and $Q=2.10^{8}$ ) [5],

- NC with energy storage cavity (ESC), scaling of ARES cavity $(R / Q=16.2 \Omega$ and $Q=105000)$ [6].

Figure 2 summarizes our best results in terms of lifetime improvement and minimization of the beam phase transients for each configuration. The extremities of the vertical bars and the marker in-between give the maximum, the minimum and the average increase factor of the lifetime, respectively. Standard ALS parameters and a $17 \%$ gap in the fill pattern were assumed.

For beam driven passive cavities, the average increase factor of the lifetime is below 1.5 with NC harmonic cavities. For passive SC and ESC cavities, a higher average increase factor can be reached, however it is still below the value of 2.2 obtained with an uniform fill and $3 \mathrm{NC}$ cavities. The ESC technology yields high average lifetime increase with still small beam phase transients. The computations show that working points with a larger phase transient generally also exhibit a larger dispersion in lifetime along the bunch train.

The main interest to use an active harmonic system is to reach the conditions for maximum bunch lengthening independently from the beam current. It is worth noting that even for active cavities, substantial phase transients are observed with a $17 \%$ gap in the fill at $400 \mathrm{~mA}$. For the
NC case, the external harmonic generator allows to achieve smaller beam phase transients and to obtain a better improvement in lifetime than for passive operation. In the case of the SC cavity the external generator does not allow to reduce the transient effects significantly. Moreover, as a SC cavity can still produce large harmonic voltage at even low currents, it turns out that there is no interest in the implementation of an external harmonic generator. Active ARES type harmonic cavities give the highest average lifetime improvement that can be obtained with a $17 \%$ gap at $400 \mathrm{~mA}$ ( factor 2.1).

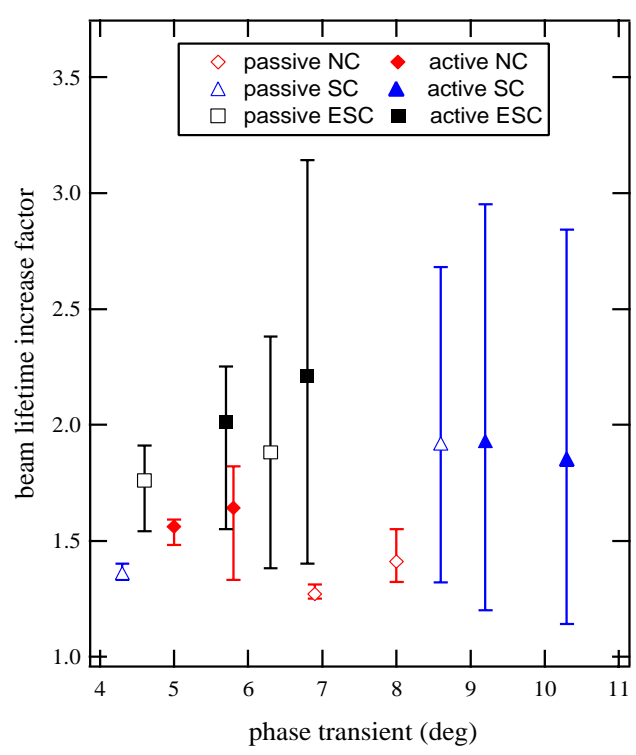

Figure 2: lifetime improvement vs phase transient for various harmonic RF system

In order to explain these results, the influence of the total $R / Q$ and the $Q$ of the harmonic system has been analyzed by comparing the results for a SC cavity with two NC cavities in table 1 . Their tuning has been varied so as to produce comparable harmonic voltages at the given beam current of $400 \mathrm{~mA}$, and a $17 \%$ gap has again been assumed. Except for a voltage of $428 \mathrm{kV}$ which corresponds to overstretching, we observe that a SC and a NC cavity with the same $R / Q=87 \Omega$ induce almost the same phase transients (phtr). It is not significantly affected by a change in Q as large as a factor $10^{4}$. With an $R / Q=3 \times 87 \Omega$, the phase transients are increased by factor varying between 2 and 4 . So, the lower the $R / Q$, the less the beam will suffer from transient effects. With respect to phase transients, SC technology has the great advantage that only one harmonic cavity is enough to provide the necessary harmonic voltage and that the total installed $R / Q$ can be several times smaller than for a standard NC solution.

\subsection{Transient compensation with beam}

Since most synchrotron light users are not sensitive to variations of the bunch intensity along the train, we have investigated the effects of a tailoring on the fill pattern to 


\begin{tabular}{|c|c|c|c|}
\hline & SC & NC & NC \\
& $\mathrm{R} / \mathrm{Q}=87 \Omega$ & $\mathrm{R} / \mathrm{Q}=87 \Omega$ & $\mathrm{R} / \mathrm{Q}=3 * 87 \Omega$ \\
\hline$V_{\text {harm }}(\mathrm{kV})$ & phtr $(\mathrm{rad})$ & phtr $(\mathrm{rad})$ & phtr $(\mathrm{rad})$ \\
\hline 430 & 0.28 & 0.6 & 0.55 \\
380 & 0.2 & 0.24 & 0.46 \\
340 & 0.15 & 0.15 & 0.4 \\
230 & 0.09 & 0.1 & 0.25 \\
170 & 0.07 & 0.07 & 0.27 \\
\hline
\end{tabular}

Table 1: comparison of transients from passive normal conducting and superconducting cavities

partially compensate for the beam loading transients. The idea was to concentrate the phase transients over a smaller number of bunches while keeping the others at the optimal phase as much as possible.

Figure 3 shows the effect of adding a surplus charge to 40 bunches at the head and the tail of the train. The intensity of the remaining 192 bunches in the middle has to be reduced to keep the total current at $400 \mathrm{~mA}$. The corresponding bunch phase function is flat in the middle of the train.

Since the Touschek lifetime is inversely proportional to each bunch charge, it is obvious to expect a reduction in the lifetime for the higher current bunches. This is true for only part of them, as shown in Fig. 3c, and overall it is more than compensated by the increased lifetime for all the bunches in the middle of the train. The average lifetime improvement is 2.09 , at least as long as the fill pattern is not too much affected by the faster decay of head and tail.

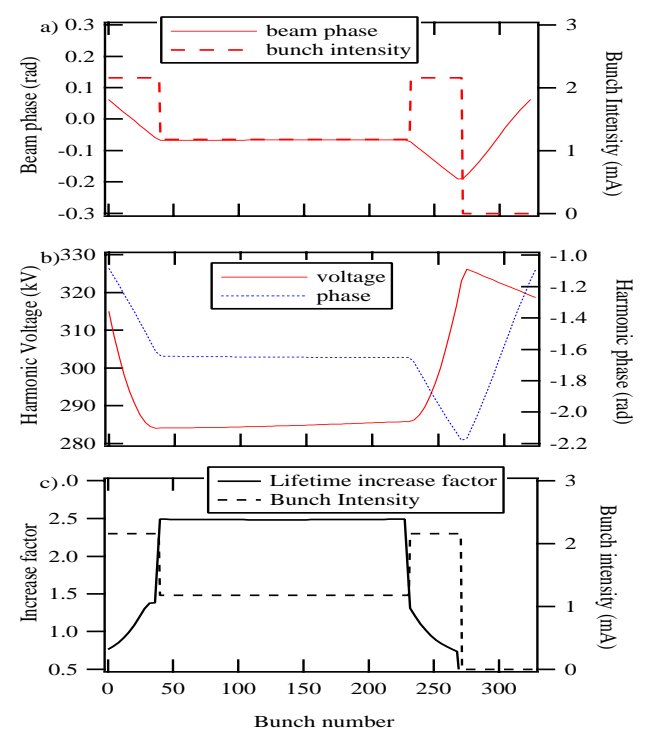

Figure 3: a) Beam phase (difference from nominal synchronous phase) and bunch intensity along the bunch train. b) Harmonic voltage and phase. c) Resulting lifetime increase with a flat maximum near the middle of the bunch train.

\section{CONCLUSIONS}

Phase transients and subsequent differential lengthening within the bunch train have been computed for various types of active and passive harmonic cavities by means of a tracking simulation. It has been shown that the adverse transient effects increase with the total $R / Q$ of the harmonic system. Normal conducting ARES systems using energy storage cavities to minimize the $R / Q$ yield the highest average lifetime improvement in the presence of a gap in the fill pattern. However, several cells would still be required to achieve the desired voltage. In general, superconducting harmonic cavities are less sensitive to transients than standard normal conducting cavities. An alternative promising method of compensating for the beam loading transients appears to be tailored fill pattern. Our example of a $50 \%$ current increase at both ends of the bunch train appears to flatten the harmonic phase over a large fraction of the bunch train and provides the best lifetime increase. There may be even better solutions. We are still investigating whether these gains are possible given practical considerations such as the achievable fill uniformity.

For normal conducting systems, an external generator has two advantages. It allows bunch lengthening down to low beam currents and helps reducing the impact of phase transients on the gain in lifetime. However, for large beam loading, an unreasonable amount of generator power is needed to cancel the transients. For a superconducting cavity, we believe a generator is of no interest.

\section{ACKNOWLEDGEMENTS}

We would like to thank Bob Rimmer for proposing the ARES structure and for many useful discussions as well as the ALS and the ESRF for supporting this work.

\section{REFERENCES}

[1] F. Pedersen," A novel RF cavity tuning feedback scheme for heavy beam loading", IEEE Trans. Nucl. Sci., NS-32, 2138, (1985). F. Pedersen, "Beam loading in the CERN PS booster", IEEE Trans. Nucl. Sci., NS-22, 1906, (1975).

[2] A. Hofmann and S. Myers, "Beam dynamics in a double RF system", Proc. of the 11th Int. Conf. on High Energy Acc. ISRTH-RF/80-26 (1980).

[3] J.M. Byrd, S. De Santis,J. Jacob,V. Serriere, ” Transient beam loading effects in harmonic RF systems for light source", submitted for publication in PRST-AB.

[4] J. M. Byrd, K. Baptiste, S. De Santis, S. Kosta, C. C. Lo, D. Plate, R. A. Rimmer, M. Franks " Design of a higher harmonic RF system for the Advanced Light Source", Nucl. Inst. Meth. A (439) 1(1999) pp. 15-25.

[5] M.Svandrlik et al., "The Super-3HC project: an idle superconducting harmonic cavity for bunch length manipulation.", Proc. of the 2000 Eur. Part. Acc. Conf., (2000).

[6] T.Kageyama et al., " The ARES cavity for the KEK-B factory”, Proc. of the 1996 Eur. Part. Acc. Conf., (1996). 